\title{
Efficient 3D-RSS map estimation method based on area classification
}

\author{
Azusa Danjo ${ }^{1 a)}$, Shingo Ata ${ }^{1}$, and Takahiro Matsuda ${ }^{2}$ \\ ${ }^{1}$ Graduate School of Engineering, Osaka City University \\ 3-3-138 Sugimoto, Sumiyoshi-ku, Osaka 558-8585, Japan \\ ${ }^{2}$ Graduate School of Systems Design, Tokyo Metropolitan University \\ 6-6 Asahiga-oka, Hino, Tokyo, 191-0065 Japan \\ a)danjo@c.info.eng.osaka-cu.ac.jp
}

\begin{abstract}
We consider the determination of three-dimensional maps of received signal strength (3D-RSS maps) for disaster-recovery networks enabled by unmanned aerial vehicles (UAVs). In this paper, we extend the existing tensor completion based estimator to propose an efficient new 3D-RSS map estimator. To reduce the sensing route length for the UAV, the proposed method utilizes two approaches for estimating the RSS maps (the tensor completion-based and path-lossbased approaches), depending upon the number of high buildings. We show by simulation experiments that the proposed method can achieve a data-collection time comparable to those of existing methods with a shorter sensing route.
\end{abstract}

Keywords: Received signal strength, Tensor completion, Unmanned aerial vehicles

Classification: Wireless communication technologies

\section{References}

[1] A. Danjo, S. Hara, T. Matsuda, F. Ono and R. Miura, "3D determination of message collection and delivery locations for UAV-enabled disaster recovery networks," IEEE Access, 2021. DOI:10.1109/ACCESS.2021. 3065698

[2] M Series, ITU 2135-1, "Guidelines for evaluation of radio interface technologies for IMT-advanced," 2009.

[3] T. Y. Ji, T. Z. Huang, X. L. Zhao, T. H. Ma, and G. Liu, "Tensor completion using total variation and low-rank matrix factorization," Inf. Sci., vol. 326, pp. 243-257, Jan. 2016. DOI:10.1016/j.ins.2015.07.049

[4] MATLAB, The MathWorks, Inc. https://www.mathworks.com/.

[5] EEM-RTM, EEM Inc., http://www.e-em.co.jp/rtm/index.html.

[6] IEEE Standard for Information technology - Telecommunications and information exchange between systems Local and metropolitan area networks - Specific requirements - Part 11: Wireless LAN Medium Access Control (MAC) and Physical Layer (PHY) specifications, IEEE Std 802.11-2016 (Revision of IEEE Std 802.11-2012), Dec. 2016. DOI:10.1109/IEEESTD. 2016.7786995 


\section{Introduction}

Unmanned aerial vehicles (UAVs) are promising for supporting existing terrestrial information networks since they can equipped with wireless communication tools and fly anywhere. In [1], we proposed a message collection and delivery system for UAV-enabled disaster-recovery networks based on three-dimensional maps of received signal strength (3D-RSS maps). The 3DRSS maps are constructed from sparsely sensed RSS using tensor completion. In [1], we found that RSS maps without any missing stationary points are essential for complete message delivery, while the sensing obviously requires the overhead of measuring many RSSs prior to message delivery. Note that this paper does not target adaptive line-of-sight (LOS) and non-line-ofsight (NLOS) environments selection. Instead, we use RSS maps to determine the UAV locations because previous works have shown that it is difficult to achieve dependable communication by considering either LOS or NLOS links alone. Therefore we use the RSS map derived from the sensed data of the received signals, which include both LOS- and NLOS-related factors.

In this paper, we propose an efficient new 3D-RSS map-estimation method by extending the existing method developed in [1]. Our proposed new method classifies 3D-RSS maps into path loss (PL)-type and tensor completion (TC)type RSS maps, according to the number of high buildings around the ground stations. We show by simulation experiments that the proposed method can achieve a data-collection time comparable to the existing methods but with a shorter sensing route.

\section{System model and assumptions}

Fig. 1 shows the system model, which involves $P$ stationary transceivers (TRXs), a 3D region over them, and a UAV flying in the region. The region has the size $X \times Y \times Z\left(\mathrm{~m}^{3}\right)$, and it is divided into $N_{1} \times N_{2} \times N_{3}=N_{\mathrm{v}}$ voxels of size $\Delta X \times \Delta Y \times \Delta Z\left(\mathrm{~m}^{3}\right)$. We define the location of the center of gravity of the voxel to be $\mathbf{g}_{i j k}=\left[x_{i}, y_{j}, z_{k}\right]^{\top}$, which we refer to as a point. In addition, we define the location of the $q$ th TRX to be $\mathbf{t}^{(p)}=\left[t_{x}^{(p)}, t_{y}^{(p)}, t_{z}^{(p)}\right]^{\top}$, and we form the set of indexes for all the TRXs: $\mathbf{T}=\{1,2, \ldots, P\}$. The $P$ TRXs transmit their wireless signals to a UAV. To sense the RSS, the UAV visits a number of voxels along a predetermined route $\Omega$.

A 3D-RSS map is defined for each TRX, and a 3D tensor can be defined for the RSS map of the $p$ th TRX as follows:

$$
\tilde{\mathcal{R}}^{(p)}=\left\{R_{i j k}^{(p)} \mid 1 \leq i \leq N_{1}, 1 \leq j \leq N_{2}, 1 \leq k \leq N_{3}\right\},
$$

where $R_{i j k}^{(p)}$ denotes the RSS value at point $\mathbf{g}_{i j k}$ from the $p$ th TRX. Note that in $\tilde{\mathcal{R}}^{(p)}$, it is not necessary to consider RSS values at voxels far from $\mathbf{t}^{(p)}$. Therefore, we consider the subset $\mathcal{R}^{(p)} \subset \tilde{\mathcal{R}}^{(p)}$, which includes only the RSS values at voxels around the given TRX. 


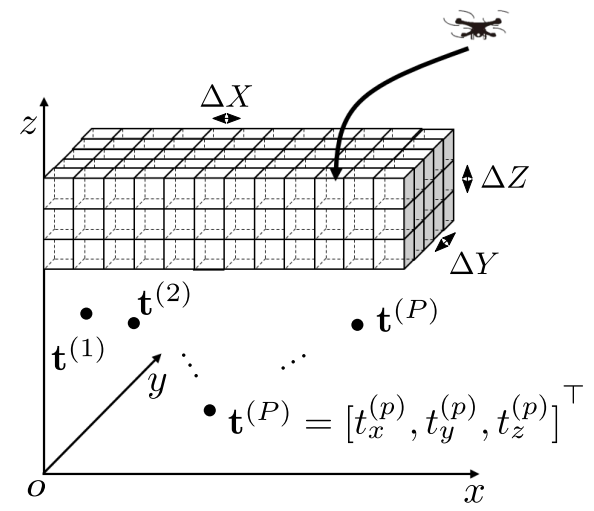

Fig. 1. System model.

\section{Determination of UAV locations necessary to collect and deliver} messages [1]

The UAV locations at which the UAVs need to be positioned to collect and deliver messages, which are hereafter referred to as message points, are determined from the estimated 3D-RSS maps. We define $\mathbf{w}_{q}(q=1,2, \ldots, Q$, $Q \leq P)$ as message points.

Let $\hat{\mathcal{R}}^{(p)}$ denote an estimated RSS map of $\mathcal{R}^{(p)}$. Using the normalized RSS correlation, we decompose $\mathbf{T}$ into the subsets $\mathbf{T}_{q}$, where $\mathbf{T}=\bigcup_{q=1}^{Q} \mathbf{T}_{q}$. Defining the RSS value for given $\mathbf{w}_{q}$ and $\mathbf{t}^{(p)}$ as $R\left(\mathbf{w}_{q}, \mathbf{t}^{(p)}\right)(\mathrm{dBm}), \mathbf{w}_{q}$ is determined by the following optimization problem:

$$
\max _{\mathbf{w}_{q}} \min _{p \in \mathbf{T}_{q}} R\left(\mathbf{w}_{q}, \mathbf{t}^{(p)}\right) .
$$

Interested readers are referred to [1] for details.

\section{3D-RSS map-estimation and sensing point determinations}

\subsection{RSS map-estimation and route establishment}

The proposed method estimates the RSS maps using a combination of two different estimators: the PL estimator and the TC estimator. Let

$$
\mathcal{R}^{(\text {all })}=\left\{\mathcal{R}^{(p)} \mid p=1,2, \ldots, P\right\}
$$

denote the set of all the 3D-RSS maps. Each 3D-RSS map is classified into either a PL-type RSS map or a TC-type RSS map based on the area classification described in section 4.2 below. We represent the set of PL-type RSS maps by $\mathcal{R}_{\mathrm{PL}} \subseteq \mathcal{R}^{(\text {all })}$ and the set of TC-type RSS maps by $\mathcal{R}_{\mathrm{TC}} \subseteq \mathcal{R}^{(\text {all })}$.

A PL-type RSS map $\mathcal{R}_{\mathrm{PL}}^{(p)} \in \mathcal{R}_{\mathrm{PL}}$ is estimated in [2] using a path-loss model. Let $\mathbf{g}=[x, y, z]^{\top}$ denote the location of a voxel in $\mathcal{R}_{\mathrm{PL}}^{(p)}$. The PL $L\left(\mathbf{p}^{(p)}, \mathbf{g}\right)$ between $\mathbf{p}^{(p)}$ and $\mathbf{g}$ is given by

$$
L\left(\mathbf{t}^{(p)}, \mathbf{g}\right)=22 \log _{10}\left(d\left(\mathbf{t}^{(p)}, \mathbf{g}\right)\right)+28+2.0 \log _{10}\left(f_{c}\right),
$$

where $d\left(\mathbf{t}^{(p)}, \mathbf{g}\right)$ denotes the distance between the UAV and the TRX, and $f_{c}$ denotes the carrier frequency. The RSS value corresponding to $\mathbf{g}$ is given by

$$
P_{\mathrm{T}}-L\left(\mathbf{t}^{(p)}, \mathbf{g}\right),
$$




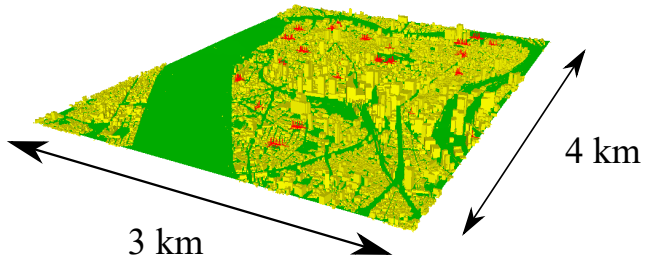

(a) 3D city map in the simulation experiments

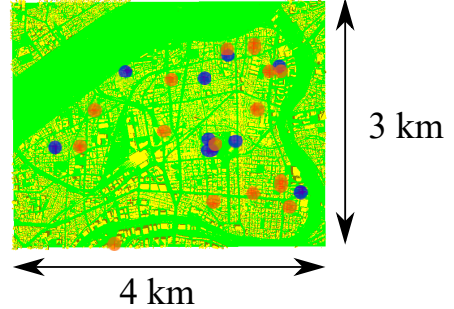

(b) Result of area classification

Fig. 2. City maps

where $P_{\mathrm{T}}$ denotes the transmission power.

On the other hand, in order to estimate the TC-type RSS maps, the UAV visits $M$ sensing points along route $\Omega$ and senses RSSs for TRXs at each sensing point. $\mathcal{R}^{(p)}$ is estimated from the $M$ RSS values with TC. As in [1], we use the total variation low rank tensor completion method [3]. It is worth mentioning that the length of $\Omega$ is reduced when more RSS maps are classified as PL-type RSS maps. In this paper, $\Omega$ is obtained using the genetic algorithm solver in MATLAB [4].

\subsection{Area classification}

When there are few high buildings around a TRX, the wireless channels between the TRX and the UAV are very likely to be LOS channels, which means that the 3D-RSS map for the TRX can be classified as a PL-type RSS map. On the other hand, when there are high buildings, the 3D-RSS map can be classified as a TC-type RSS map, because the wireless channels are likely to be NLOS channels.

Therefore, it is reasonable to classify the RSS maps based on the heights of the surrounding buildings. We define the number of buildings higher than $\rho_{f}$ in a RSS construction area for the $p$ th TRX as $N^{(p)}$. The RSS map $\mathcal{R}^{(p)}$ is classified as a TC-type RSS map if $N^{(p)}>N_{\text {th }}$, and it is classified as a PL-type RSS map otherwise, where $N_{\text {th }}$ denotes the threshold value for $N^{(p)}$.

\section{Performance evaluation}

To evaluate the performance of the proposed method, we conducted simulation experiments. Fig. 2(a) shows the 3D city map used for the experiments, where 123 TRXs are deployed. Fig. 2(b) shows the result obtained by applying the area classification method described above. We obtained the RSS values by using the ray-tracing wireless-propagation simulator EEM-RTM [5], which uses the RSS values as the ground truth for the 3D-RSS maps.

We validated the proposed method, which we term the AC method, by comparing it with two other methods: the TC method and the PL method. In the TC method, all the RSS maps are classified as TC-type RSS maps, on the other hand, in the PL method, they are classified as PL-type RSS maps. Note that the performance of the TC method is obtained by setting $\rho_{f}=0$ while that of the PL method is obtained by setting $\rho_{f}=\infty$. 
To evaluate the data collection time, we defined the message collection time between the $p$-th TRX and the $q$-the message point as

$$
T_{\mathrm{c}}\left(\mathbf{w}_{q}, \mathbf{t}^{(p)}\right)=D / f\left(R\left(\mathbf{w}_{q}, \mathbf{t}^{(p)}\right)\right),
$$

where $D=100(\mathrm{MB})$ denotes the size of the message, and $f\left(R\left(\mathbf{w}_{q}, \mathbf{t}^{p}\right)\right)$ (Mbps) denotes the transmission rate, which is defined as [6]

$$
f\left(R\left(\mathbf{w}_{q}, \mathbf{t}^{(p)}\right)\right)=\left\{\begin{array}{rl}
6.5 & \left(-82 \leq R\left(\mathbf{w}_{q}, \mathbf{t}^{(p)}\right)<-79\right) \\
13.0 & \left(-79 \leq R\left(\mathbf{w}_{q}, \mathbf{t}^{(p)}\right)<-77\right) \\
19.5 & \left(-77 \leq R\left(\mathbf{w}_{q}, \mathbf{t}^{(p)}\right)<-74\right) \\
26.0 & \left(-74 \leq R\left(\mathbf{w}_{q}, \mathbf{t}^{(p)}\right)<-70\right) \\
39.0 & \left(-70 \leq R\left(\mathbf{w}_{q}, \mathbf{t}^{(p)}\right)<-66\right) \\
52.0 & \left(-66 \leq R\left(\mathbf{w}_{q}, \mathbf{t}^{(p)}\right)<-65\right) \\
58.5 & \left(-65 \leq R\left(\mathbf{w}_{q}, \mathbf{t}^{(p)}\right)<-64\right) \\
65.0 & \left(-64 \leq R\left(\mathbf{w}_{q}, \mathbf{t}^{(p)}\right)\right)
\end{array} .\right.
$$

Fig. 3(a) shows the dependence of the AC method on $\rho_{f}$. The horizontal axis represents the threshold of the number of floors, and the vertical axis is gives the length of the sensing route and the total data-collection time $T_{c}^{(\text {all })}=$ $\sum_{p=1}^{P} T_{\mathrm{c}}\left(\mathbf{w}_{q}, \mathbf{t}^{(p)}\right)$ for the AC method, normalized by the corresponding values for the TC method. The total length of the sensing route for the AC method decreases as $\rho_{f}$ increases. However, when $\rho_{f}$ is 11 or higher, the total datacollection time for the AC method becomes infinite, which means that some TRXs have failed to communicate. Because we require $100 \%$ coverage of the TRXs, $\rho_{f}$ must therefore be equal to or less than 10. When $\rho_{f}=10$, with only a $12 \%$ increase in the data-collection time, the length of the sensing route can be reduced by $60 \%$. Note that the threshold is determined by the environment, and the determination of that quantity will be considered in future work.

Fig. 3(b) compares the empirical cumulative distribution function (ECDF) of $T_{\mathrm{c}}\left(\mathbf{w}_{q}, \mathbf{t}^{(p)}\right)$ among the TC, PL, and AC methods, where $\rho_{f}=10$. This figure shows that the distribution of the data-collection time for the PL method has a longer tail than for either the AC or TC methods. Eqn. (7) shows that the transmission rate does not increase linearly as the RSS values increase, and by using the RSS map the UAVs can construct an appropriate path by selecting areas that have high RSS values. As a result, the variation of RSS values among the TRXs becomes small, which enables a stable data-transfer rate. On the other hand, the RSS from every TRX may vary randomly in a LOS environment, and the RSS values may be distributed more widely in the PL method, so there can be cases with worse transmission rates. In addition, The ECDF does not converge to 1 in the PL method, which means that some message points have infinite data collection times. The reason is that the RSS values are not correctly estimated by using only the PL model.

\section{Conclusion}

In this paper, we have proposed a new 3D-RSS map-estimation method for 


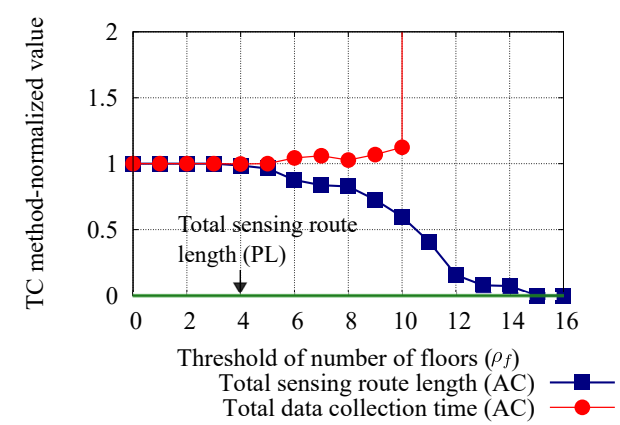

(a) Dependency of the TC method-normalized total sensing route length and total data collection time for AC method on $\rho_{f}$.

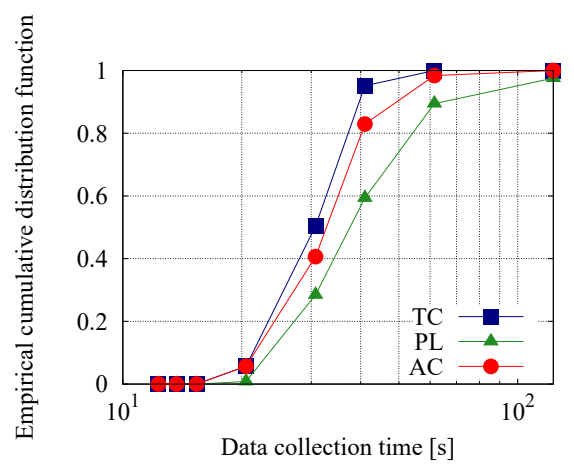

(b) Empirical cumulative distribution function of data collection time

Fig. 3. Simulation results.

RSS maps as TC-type and PL-type RSS maps, depending upon the number of high buildings around the corresponding TRXs. Performance evaluation by simulation experiments showed that the proposed method can reduce the length of the sensing route without increasing the total data collection time.

\section{Acknowledgments}

This work was supported in part by the Grants-in-Aid for Scientific Research (B) from Japan Society for the Promotion of Science (JSPS) under Grant JP19H04096, and TMU local 5G research support. 